TAIWANESE JOURNAL OF MATHEMATICS

Vol. 14, No. 3B, pp. 1047-1053, June 2010

This paper is available online at http://www.tjm.nsysu.edu.tw/

\title{
LINEAR ORTHOGONALITY PRESERVERS OF STANDARD OPERATOR ALGEBRAS
}

\author{
Chung-Wen Tsai and Ngai-Ching Wong \\ Dedicated to the Memory of Professor Sen-Yen Shaw
}

\begin{abstract}
In 2003, Araujo and Jarosz showed that every bijective linear map $\theta: A \rightarrow B$ between unital standard operator algebras preserving zero products in two ways is a scalar multiple of an inner automorphism. Later in 2007, Zhao and Hou showed that similar results hold if both $A, B$ are unital standard algebras on Hilbert spaces and $\theta$ preserves range or domain orthogonality. In particular, such maps are automatically bounded. In this paper, we will study linear orthogonality preservers in a unified way. We will show that every surjective linear map between standard operator algebras preserving range/domain orthogonality carries a standard form, and is thus automatically bounded.
\end{abstract}

\section{INTRODUCTION}

An algebra $A$ of bounded linear operators on a Banach space $M$ is called standard if $A$ contains the algebra $\mathcal{F}(M)$ of all bounded finite rank operators on $M$. Assume that $\theta: A \rightarrow B$ is a bijective linear map between two unital standard operator algebras on Banach spaces $M, N$, preserving zero products in two ways, i.e., $a b=0$ in $A$ if and only if $\theta(a) \theta(b)=0$ in $B$. Araujo and Jarosz [1] showed that in this case there exist a nonzero scalar $\lambda$ and a bounded invertible linear map $S: M \rightarrow N$ such that

$$
\theta(a)=\lambda S a S^{-1}, \quad \forall a \in A .
$$

It was pointed out in [3] that the same result holds also when $A, B$ are non-unital.

On the other hand, let $A, B$ be unital standard operator algebras on (real or complex) infinite dimensional Hilbert spaces $H, K$, respectively. Assume that $\theta$ :

Received November 8, 2009.

2000 Mathematics Subject Classification: 46J10, 46L05.

Key words and phrases: Linear orthogonality preservers, Standard operator algebras, Autocontinuity. This work is partially supported by Taiwan National Science Council grants 96-2115-M-110-004-MY3. 
$A \rightarrow B$ is a surjective additive map preserving range orthogonality in two ways, i.e., $a^{*} b=0$ in $A$ if and only if $\theta(a)^{*} \theta(b)=0$ in $B$. Zhao and Hou [6] showed that in this case there exist a unitary (or conjugate unitary) operator $U: H \rightarrow K$ and a bounded linear (or conjugate linear) invertible operator $V: K \rightarrow H$ such that

$$
\theta(a)=U a V, \quad \forall a \in A .
$$

Zhao and Hou [6] also obtained a similar version for surjective additive maps preserving domain orthogonality in two ways, i.e., the ones with $\theta(a) \theta(b)^{*}=0$ exactly when $a b^{*}=0$.

In this paper, we will give a unified approach, with new proofs, to different linear orthogonality preservers. We will show that every surjective linear map $\theta$ : $A \rightarrow B$ between two standard operator algebras on (real or complex) Hilbert spaces preserving range/domain orthogonality in two ways carries a standard form, and is thus automatically bounded as well. The following table summarizes our results.

\begin{tabular}{cc}
\hline The structures $\theta$ preserves & The form of $\theta$ carries \\
\hline$a b=0 \Leftrightarrow \theta(a) \theta(b)=0$ & $\lambda S a S^{-1}$ \\
$a^{*} b=0 \Leftrightarrow \theta(a)^{*} \theta(b)=0$ & $U a T$ \\
$a b^{*}=0 \Leftrightarrow \theta(a) \theta(b)^{*}=0$ & $S a V$ \\
$a^{*} b=0 \Leftrightarrow \theta(a) \theta(b)^{*}=0$ & $S a^{t} V$ \\
$a b^{*}=0 \Leftrightarrow \theta(a)^{*} \theta(b)=0$ & $U a^{t} T$ \\
$a^{*} b=a b^{*}=0 \Leftrightarrow \theta(a)^{*} \theta(b)=\theta(a) \theta(b)^{*}=0$ & $\lambda U a V$ or $\lambda U a^{t} V$ \\
\hline$\theta \quad:$ a surjective linear map between standard operator algebras \\
$\lambda \quad:$ a nonzero scalar \\
$S, T:$ bounded invertible linear operators \\
$U, V:$ unitary operators
\end{tabular}

We note that we need to assume $A$ is unital or $A$ contains all trace class operators in the second to the fifth cases. Without this assumption, $\theta$ can be unbounded. For example, let $H$ be an infinite dimensional Hilbert space and let $T$ be any unbounded bijective linear operator on $H$. Then $x \otimes y \mapsto x \otimes T y$ (resp. $x \otimes y \mapsto T x \otimes y$ ) defines an unbounded bijective range (resp. domain) orthogonality preserving linear map from $\mathcal{F}(H)$ onto $\mathcal{F}(H)$. However, in the first case of zero product preservers and in the last case of doubly orthogonality preservers, this assumption can be dropped.

\section{PReLIMINARIES}

In the following, $A$ and $B$ are standard operator algebras on (real or complex) Hilbert spaces $H, K$, respectively, and $\theta$ is a surjective linear map from $A$ onto $B$. 
As pointed out in [6], if $\theta$ preserves any kind of orthogonality in two ways, then $\theta$ is injective. For example, if $\theta(x)=0$ and $\theta$ preserves zero products in two ways, then $\theta(x) \theta(y)=0$ implies $x y=0$ for all $y$ in $A$. Thus $x=0$ as well.

Recall that by the Fundamental Theorem of Affine Geometry any bijective linear map $\theta: \mathcal{F}(H) \rightarrow \mathcal{F}(K)$ sending exactly rank one operators onto rank one operators must be in one of the following forms.

(1) $\theta(x \otimes y)=S x \otimes R y$, where $S, R: H \rightarrow K$ are invertible linear maps.

(2) $\theta(x \otimes y)=R y \otimes S x$, where $S, R: H \rightarrow K$ are invertible conjugate linear maps.

Here, $x \otimes y(z)=\langle z, y\rangle x$ is the rank at most one operator, and $\langle\cdot, \cdot\rangle$ is the inner product of the Hilbert space $H$ or $K$. Note that for any scalar $\alpha$ we have $\alpha(x \otimes y)=$ $(\alpha x) \otimes y=x \otimes(\bar{\alpha} y)$. See, e.g., $[4,6]$.

Fix an orthonormal basis $\left\{e_{j}\right\}$ of a Hilbert space $H$. For all $x=\sum\left\langle x, e_{j}\right\rangle e_{j}$ in $H$, we set $\bar{x}=\sum\left\langle e_{j}, x\right\rangle e_{j}$. Let $T$ be a bounded linear operator on $H$. The transpose operator $T^{t}$ of $T$ with respect to $\left\{e_{j}\right\}$ is the bounded linear operator satisfying the condition

$$
\left\langle T e_{i}, e_{j}\right\rangle=\left\langle e_{i}, T^{*} e_{j}\right\rangle=\left\langle T^{t} e_{j}, e_{i}\right\rangle, \quad \forall i, j .
$$

The transpose operator is well-defined and $\|T\|=\left\|T^{*}\right\|=\left\|T^{t}\right\|$. Here $T^{*}$ is the adjoint operator of $T$. Note that the definition of $\bar{x}$ and $T^{t}$ depend on the choice of the orthonormal basis. However, they are unique up to unitarily equivalence.

Some properties of the transpose operators are given below. For all $x, y \in H$ we have

(1) $\langle\bar{x}, \bar{y}\rangle=\langle y, x\rangle$.

(2) $(x \otimes y)^{t}=\bar{y} \otimes \bar{x}$.

(3) $\left(T^{t}\right)^{*}=\left(T^{*}\right)^{t}$.

(4) $T^{t} x=\overline{T^{*} \bar{x}}$.

\section{RESULTS}

We first give, with a new proof, a modified version of the result of Zhao and Hou in [6] about linear range orthogonality preservers mentioned in the introduction. Note that we can allow the algebras not being unital, provided instead that they contain trace class operators.

Theorem 1. Let $A, B$ be standard operator algebras on Hilbert spaces $H, K$, respectively. Suppose $A$ is unital, or $A$ contains all trace class operators on $H$. Assume that $\theta: A \rightarrow B$ is a surjective linear map such that $a^{*} b=0$ if and only if $\theta(a)^{*} \theta(b)=0$. Then $\theta$ is bounded, and there exist a bounded invertible linear operator $T: K \rightarrow H$ and a unitary operator $U: H \rightarrow K$ such that

$$
\theta(a)=U a T, \quad \forall a \in A .
$$


Proof. Note that $\theta$ is indeed bijective. Put

$$
a^{\dashv}=\left\{c \in A: c^{*} a=0\right\}, \quad \text { for all nonzero } a \text { in } A .
$$

For any $a$ and $b$ in $A$, it is clear that $a^{\dashv} \subseteq b^{\dashv}$ if and only if the closure of the range space of $a$ contains that of $b$. We define a partial order on $A$ by $a \leq b$ if and only if $a^{\dashv} \subseteq b^{\dashv}$. In this partial order, $a$ is a maximum if and only if $a$ is a rank one operator. By the two way range orthogonality preserving assumption, we see that both $\theta$ and $\theta^{-1}$ preserve this partial order, and thus send the maxima onto the maxima. In other words, $\theta$ and $\theta^{-1}$ send rank one operators onto rank one operators. It then follows from the Fundamental Theorem of Affine Geometry that there exist invertible linear or conjugate linear maps $S: H \rightarrow K$ and $R: K \rightarrow H$ such that either

$$
\theta(x \otimes y)=S x \otimes R y, \quad \forall x, y \in H,
$$

or

$$
\theta(x \otimes y)=S y \otimes R x, \quad \forall x, y \in H .
$$

However, the second case does not give us a range orthogonality preserver, and thus be ruled out.

Observe that

$$
\begin{array}{lll} 
& \left\langle x_{1}, x_{2}\right\rangle=0 & \\
\text { implies } & \left(x_{2} \otimes y_{2}\right)^{*}\left(x_{1} \otimes y_{1}\right)=0, & \forall y_{1}, y_{2} \in H \\
\text { implies } & \theta\left(x_{2} \otimes y_{2}\right)^{*} \theta\left(x_{1} \otimes y_{1}\right)=0, & \forall y_{1}, y_{2} \in H \\
\text { implies } & \left(S x_{2} \otimes R y_{2}\right)^{*}\left(S x_{1} \otimes R y_{1}\right)=0, & \forall y_{1}, y_{2} \in H \\
\text { implies } & \left\langle S x_{1}, S x_{2}\right\rangle=0 . &
\end{array}
$$

For any two orthogonal norm one elements $x, y$ in $H$, we have $\langle x, y\rangle=\langle x+$ $y, x-y\rangle=0$. This gives $\langle S x, S y\rangle=\langle S x+S y, S x-S y\rangle=0$, and therefore $\|S x\|=\|S y\|$. It follows that $S=\lambda U$ for a nonzero scalar $\lambda$ and a unitary operator $U$ from $H$ onto $K$. Renaming $\lambda R$ by $R$, we will have

$$
\theta(x \otimes y)=U x \otimes R y, \quad \forall x, y \in H .
$$

To get the boundedness of $R$ we need to utilize the extra assumptions on $A$ now. Suppose first that $A$ is unital. For any norm one element $e$ in $H$, as $(e \otimes e)(1-$ $e \otimes e)=0$, we have $\theta(e \otimes e)^{*}(\theta(1)-\theta(e \otimes e))=0$. It follows $R e \otimes \theta(1)^{*} U e=$ $\langle U e, U e\rangle R e \otimes R e=R e \otimes R e$, and consequently, $R e=\theta(1)^{*} e$. So $R=\theta(1)^{*} U$ is bounded.

Suppose then that $A$ contains all trace class operators on $H$ and $H$ is of infinite dimension. Suppose on contrary that there were an orthonormal sequence $\left\{x_{n}\right\}$ in 
$H$ such that $\left\|R x_{n}\right\| \geq n^{3}$ for $n=1,2,3, \ldots$. Define a trace class operator $W$ on $H$ by $W=\sum_{n} x_{n} \otimes x_{n} / n^{2}$. Since $\left(x_{n} \otimes x_{n}\right)\left(n^{2} W-x_{n} \otimes x_{n}\right)=0$, we have $\theta\left(x_{n} \otimes x_{n}\right)^{*}\left(n^{2} \theta(W)-\theta\left(x_{n} \otimes x_{n}\right)\right)=0$. It follows $n^{2} R x_{n} \otimes \theta(W)^{*} U x_{n}=$ $\left\langle U x_{n}, U x_{n}\right\rangle R x_{n} \otimes R x_{n}=R x_{n} \otimes R x_{n}$. As a result, $\left\|\theta(W)^{*}\right\| \geq\left\|\theta(W)^{*} U x_{n}\right\|=$ $\left\|R x_{n}\right\| / n^{2} \geq n$ for all $n=1,2,3, \ldots$. This contradiction ensures again that $R$ is bounded.

Let $a \in A$. For any $x \neq 0$ in $H$, let $y \in H$ such that $\langle x, y\rangle=1$. Set $b=a-\left(y \otimes a^{*} x\right)$. Observe $b^{*}(x \otimes y)=0$. Thus,

$$
\begin{aligned}
0=\theta(b)^{*} \theta(x \otimes y) & =\left(\theta(b)^{*} U x\right) \otimes R y \\
& =\left(\left[\theta(a)^{*}-\theta\left(y \otimes a^{*} x\right)^{*}\right] U x\right) \otimes R y \\
& =\left(\theta(a)^{*} U x-\left(R a^{*} x \otimes U y\right) U x\right) \otimes R y .
\end{aligned}
$$

This implies

$$
\theta(a)^{*} U x=\left(R a^{*} x \otimes U y\right) U x=R a^{*} x, \quad \forall x \in H .
$$

Hence,

$$
\theta(a)=U a R^{*}, \quad \forall a \in A .
$$

Setting $T=R^{*}$, we are done, as the boundedness of $\theta$ is now clear.

Next, we consider the other cases $\theta$ transforming the domain/range orthogonality to the domain/range orthogonality.

Theorem 2. Let $A, B$ be standard operator algebras on Hilbert spaces $H, K$, respectively. Suppose $A$ is unital, or A contains all trace class operators on $H$. Let $\theta: A \rightarrow B$ be a surjective linear map.

(a) Assume that $a b^{*}=0$ if and only if $\theta(a) \theta(b)^{*}=0$. Then $\theta$ is bounded, and there exists a bounded invertible linear operator $S: H \rightarrow K$ and a unitary operator $V: K \rightarrow H$ such that

$$
\theta(a)=S a V, \quad \forall a \in A .
$$

(b) Assume that $a^{*} b=0$ if and only if $\theta(a) \theta(b)^{*}=0$. Then $\theta$ is bounded, and there exist a bounded invertible linear operator $S: H \rightarrow K$ and a unitary operator $V: K \rightarrow H$ such that

$$
\theta(a)=S a^{t} V, \quad \forall a \in A .
$$

(c) Assume that $a b^{*}=0$ if and only if $\theta(a)^{*} \theta(b)=0$. Then there exist a unitary operator $U: H \rightarrow K$ and a bounded invertible linear operator operator $T: K \rightarrow H$ such that

$$
\theta(a)=U a^{t} T, \quad \forall a \in A .
$$


Proof. For a fixed orthonormal basis, we can define three range orthogonality preserving surjective linear maps respectively by setting

$$
a \mapsto \theta\left(a^{t}\right)^{t}, \quad a \mapsto \theta(a)^{t}, \quad \text { and } a \mapsto \theta\left(a^{t}\right) .
$$

Then Theorem 1 applies.

Finally, we will investigate the doubly orthogonality preservers. A map $\theta$ is called a doubly orthogonality preserver if $\theta(a)^{*} \theta(b)=\theta(a) \theta(b)^{*}=0$ whenever $a^{*} b=a b^{*}=0$. Bounded doubly orthogonality preservers between $\mathrm{C}^{*}$-algebras and $\mathrm{JB}^{*}$-algebras are studied in $[5,2]$. Note also that like the case of the zero product preservers, we do not need to assume $A$ is unital or $A$ contains any trace class operator on $H$ in this case.

Theorem 3. Let $\theta: A \rightarrow B$ be a surjective linear map between standard operator algebras on Hilbert space $H, K$, respectively, such that $a^{*} b=a b^{*}=0$ if and only if $\theta(a)^{*} \theta(b)=\theta(a) \theta(b)^{*}=0$. Then $\theta$ is bounded, and there exist a nonzero scalar $\lambda$ and unitary operators $U: H \rightarrow K$ and $V: K \rightarrow H$ such that either

$$
\theta(a)=\lambda U a V, \quad \forall a \in A,
$$

or

$$
\theta(a)=\lambda U a^{t} V, \quad \forall a \in A .
$$

Proof. Put for all nonzero $a$ in $A$ that

$$
a^{\dashv}=\left\{c \in A: c^{*} a=0\right\} \quad \text { and } \quad a^{\vdash}=\left\{c \in A: a c^{*}=0\right\} .
$$

Set $a^{+}=a^{\dashv} \cap a^{\vdash}$. For any $a$ and $b$ in $A$, it is not difficult to see that that $a^{+} \subseteq b^{+}$ if and only if the closure of the range space of $a$ contains that of $b$, and the initial space of $a$ contains that of $b$. Define a partial order on $A$ by saying $a \leq b$ if and only if $a^{+} \subseteq b^{+}$. In this partial order, $a$ is a maximum if and only if $a$ is of rank one. By the doubly orthogonality preserving property of $\theta$ and $\theta^{-1}$, we see that both of them preserves this partial order, and thus sends the maxima onto the maxima. In other words, both $\theta$ and $\theta^{-1}$ send rank one operators onto rank one operators. It then follows from the Fundamental Theorem of Affine Geometry that there exist invertible linear or conjugate linear maps $S: H \rightarrow K$ and $R: K \rightarrow H$ such that either

$$
\theta(x \otimes y)=S x \otimes R y, \quad \forall x, y \in H,
$$

or

$$
\theta(x \otimes y)=R y \otimes S x, \quad \forall x, y \in H .
$$

By replacing $\theta$ with the map $a \mapsto \theta(a)^{t}$ if necessary, we can assume that the first case happens. 
Arguing as in the proof of Theorem 1, we will see that there exist nonzero scalars $\lambda_{1}, \lambda_{2}$ such that $U=\lambda_{1}^{-1} S$ is a unitary operator from $H$ onto $K$, and $W=\lambda_{2}^{-1} R$ is a unitary operator from $K$ onto $H$. Put $\lambda=\lambda_{1} \lambda_{2}$ and $V=W^{*}$, we will have

$$
\theta(a)=\lambda U a V, \quad \forall a \in \mathcal{F}(H) .
$$

In general, let $a \in A$. For any $x$ in $H$ with $a^{*} x \neq 0$, let $y \in H$ such that $\langle x, a y\rangle=1$. Set $b=a-\left(a y \otimes a^{*} x\right)$. Observe $b^{*}(x \otimes y)=b(x \otimes y)^{*}=0$. Thus,

$$
\begin{aligned}
0=\theta(b)^{*} \theta(x \otimes y) & =\lambda\left(\theta(b)^{*} U x\right) \otimes V^{*} y \\
& =\lambda\left(\left[\theta(a)^{*}-\theta\left(a y \otimes a^{*} x\right)^{*}\right] U x\right) \otimes V^{*} y \\
& =\lambda\left(\theta(a)^{*} U x-\bar{\lambda}\left(V^{*} a^{*} x \otimes U a y\right) U x\right) \otimes V^{*} y .
\end{aligned}
$$

This implies

$$
\theta(a)^{*} U x=\bar{\lambda}\left(V^{*} a^{*} x \otimes U a y\right) U x=\lambda V^{*} a^{*} x, \quad \forall x \in H .
$$

Hence,

$$
\theta(a)=\lambda U a V, \quad \forall a \in A .
$$

The map $\theta$ is clearly bounded.

\section{REFERENCES}

1. J. Araujo and K. Jarosz, Biseparating maps between operator algebras, J. Math. Anal. Appl., 282 (2003), 48-55.

2. M. Burgos, F. J. Fernández-Polo, J. J. Garcés, J. Martínez Moreno and A. M. Peralta, Orthogonality preservers in $\mathrm{C}^{*}$-algebras, $J B^{*}$-algebras and $\mathrm{JB}^{*}$-triples, J. Math. Anal. Appl., 348 (2008), 220-233.

3. C.-W. Leung and N.-C. Wong, Zero Product Preserving Linear Maps of CCR C*algebras with Hausdorff Spectrum, J. Math. Anal. Appl., 361 (2010), 187-194.

4. M. Omladic and P. Semrl, Additive mappings preserving operators of rank one, Linear Algebra Appl., 182 (1993), 239-256.

5. N.-C. Wong, Triple homomorphisms of operators algebras, Southeast Asian Bulletin of Math., 29 (2005), 401-407.

6. Liankuo Zhao and Jinchuan Hou, Additive Maps Preserving Indefinite SemiOrthogonality, Journal of Systems Science and Mathematical Sciences (in Chinese), 27(5) (2007), 697-702.

Chung-Wen Tsai and Ngai-Ching Wong

Department of Applied Mathematics,

National Sun Yat-sen University,

Kaohsiung 80424, Taiwan

E-mail: tsaicw@math.nsysu.edu.tw wong@math.nsysu.edu.tw 